\title{
Tecnologia 'Inteligente' Associada a Aprendizagem Significativa em Bioquímica
}

\author{
Adriana Cardoso Furtado ${ }^{1}$, Vandor Roberto Vilardi Rissoli ${ }^{2}$
}

Escola de Saúde e Medicina (ESM) - Curso de Farmácia

Universidade Católica de Brasília (UCB), Taguatinga, DF

Núcleo de Apoio Estratégico e Educacional (NAEEd) - Faculdade do Gama Universidade de Brasília (UnB/FGA), Gama, DF

\begin{abstract}
Apoio: Conselho Nacional de Desenvolvimento Científico e Tecnológico (CNPq) adrianacfurtado.ucbegmail.com, vandorissoliegmail.com
\end{abstract}

\begin{abstract}
This work presents the integration of an educational methodology with an interactive technology of 'intelligent' educational software in overcoming the existing challenges in teaching the discipline of Biochemistry. An experiment was carried out in an undergraduate teaching environment in the Health area and obtained promising results combining Subsumption Theory with the Intelligent Tutoring System known as SAE.
\end{abstract}

Resumo. Este trabalho apresenta a integração de uma metodologia educacional com uma tecnologia interativa de software educacional 'inteligente' na superação dos desafios existentes no ensino da disciplina de Bioquímica. Um experimento foi realizado em ambiente de ensino de graduação na área da Saúde e obteve resultados promissores combinando a Teoria da Aprendizagem Significativa com o Sistema Tutor Inteligente conhecido como SAE.

\section{Introdução}

A formação em saúde geralmente é organizada em etapas (ou fases) que envolvem conteúdos importantes a serem assimilados pelos aprendizes em suas respectivas áreas profissionais, podendo ser destacado o estudo da Bioquímica como uma das etapas relevantes e fundamentais à formação de seus diferentes profissionais (médicos, enfermeiros, nutricionistas, farmacêuticos, etc.). Sendo ela complexa, mas essencial à compreensão do funcionamento ordenado das reações químicas, tratando de suas funções, estruturas e processos metabólicos que tornam possível a vida (Albuquerque et al, 2012).

A disciplina de Bioquímica corresponde a parte integrante da formação básica na área da Saúde, sendo cursada por seus estudantes novatos. Nas instituições de ensino superior tem sido observada, nos últimos anos, a dificuldade desses estudantes em compreender os conteúdos abordados pela disciplina, principalmente naqueles que requerem conhecimentos básicos de química, independente do curso a que os alunos se destinam (Yokaichiya, Galembeck e Torres, 2004).

Outro aspecto importante está relacionado ao crescimento do conhecimento nas diversas áreas das Ciências Biológicas, em particular, na Bioquímica e na Biologia Molecular (Regis, Gonçalves e Santoro, 2001). Na prática, ainda se utiliza muito da abstração para descrever os fenômenos que acontecem em nível molecular. Por outro lado, as particularidades da bioquímica já são suficientes para reconhece-la como uma 
VIII Congresso Brasileiro de Informática na Educação (CBIE 2019)

Anais do XXV Workshop de Informática na Escola (WIE 2019)

ciência dinâmica, que demanda de métodos e técnicas condizentes à maior eficiência em seu processo educacional.

Vários são os desafios educacionais para a aprendizagem satisfatória em Bioquímica, estando entre estes a formação de turmas discentes bem heterogêneas, envolvendo estudantes de cursos diferentes (Medicina, Nutrição, Farmácia, etc.), e um volume significativo de novos conhecimentos a serem assimilados por seus aprendizes. Estas turmas, por sua vez, ainda possuem quantidades elevadas de estudantes, impedindo que seu processo educacional seja personalizado, quando efetuado de maneira mais tradicional.

Diante dessa realidade, posturas educacionais mais ativas e empenhadas na aprendizagem sob medida de cada aprendiz, precisam fazer parte das estratégias educacionais em Bioquímica, a fim de propiciar o processo de ensino que anseie pela aprendizagem respeitando as peculiaridades de seus conteúdos letivos e as características de cada um de seus aprendizes.

O uso de novas estratégias de ensino pelos professores pode colaborar na melhora do desempenho e reduzir o desinteresse dos novos aprendizes pela busca das habilidades cognitivas que lhe são necessárias. Nesse ínterim, foi aplicada a metodologia de aprendizagem conhecida como Aprendizagem Significativa (Subsumption Theory) (Ausubel, 2000). Sintonizado aos princípios da Teoria da Aprendizagem Significativa (TAS), e apoiando a aplicação das técnicas oriundas de sua metodologia, um software educacional, proveniente da área da Inteligência Artificial, foi empregado em um experimento inovador com uma turma de graduação em Bioquímica, durante um semestre letivo, envolvendo estudantes de diferentes cursos na área da Saúde.

\section{Reflexões Educacionais}

A disciplina Bioquímica aborda temas importantes como estruturas orgânicas dos seres vivos, vias metabólicas e mecanismos de regulação que fazem com que ela seja obrigatória nos cursos de graduação da área da Saúde. A bioquímica tem-se tornado cada vez mais uma das ciências que mais cresce na exploração do conhecimento humano, sendo suas averiguações relevantes na elucidação do mecanismo fisiológico e na regulação de compostos bioquímicos relevantes à saúde do ser humano (Vieira, 2003).

Apesar dos estudantes reconhecerem a importância do seu aprendizado para o exercício de sua futura profissão (Oliveira et al, 2010), também têm apontado a Bioquímica como sendo uma disciplina de nível complexo e de difícil assimilação, principalmente por ser ministrada nos semestres iniciais dos cursos (Pinheiro et al, 2009).

Em um estudo, na Universidade Federal do Piauí, em que se avaliou as dificuldades de seus discentes no aprendizado em Bioquímica, foi constatado que 86,5\% dos alunos pesquisados apresentaram um grau de dificuldade de aprendizado de médio a alto. Associado a esses dados, $81,03 \%$ dos estudantes reconheceram ter passado por dificuldades de aprendizado no ensino médio, sendo que as disciplinas mais mencionadas foram: Matemática $(44,73 \%)$ e Química $(38,98 \%)$. Como consequência desse problema, $21,4 \%$ do total dos discentes pesquisados eram repetentes na disciplina de Bioquímica, o que representava um grupo significativo de alunos com grandes dificuldades de aprendizado (de Andrade, Silva e Zierer, 2017).

Ainda foi solicitado, nessa mesma pesquisa, que os estudantes elegessem três motivos para justificar as suas dificuldades de aprendizado. Como resultado, os principais motivos citados pelos alunos foram o excesso de conceitos envolvidos no estudo da 
VIII Congresso Brasileiro de Informática na Educação (CBIE 2019)

Anais do XXV Workshop de Informática na Escola (WIE 2019)

Bioquímica (54\%), seguido do fato deles considerarem o volume de informação muito grande a ser abordado em pouco tempo $(48,2 \%)$ e a presença de outras disciplinas complexas na grade curricular de cada curso $(39,4 \%)$.

Yokaichiya, Galembeck e Torres (2004) ainda apresentam, em estudos anteriores, que mesmo em programas tradicionais que possuem a disciplina de Bioquímica organizada e coerente aos objetivos do estudo, muitos dos seus alunos a definem como uma coleção de estruturas e reações químicas de difícil assimilação, além de não serem integradas à prática profissional.

O ensino e a aprendizagem da disciplina de Bioquímica são um desafio pela própria natureza desse conhecimento, principalmente pela terminologia ampla e específica (nomes, fórmulas e estruturas químicas), que exige um alto grau de abstração e um conhecimento básico de Química (Schönborn e Anderson, 2010).

As aulas expositivas, método pedagógico mais utilizado pelos docentes, tendem a se concentrar na abordagem de conceitos e definições relevantes ao conteúdo estudado, além de expor fatos e eventos isolados que precisam ser conhecidos pelos aprendizes (Gee, 2008). No entanto, a aplicação prática desses conteúdos abordados em atividades mais sintonizadas à atuação desses profissionais fica limitada, quando emprega somente as aulas expositivas.

A ausência de ações programadas em laboratório, a falta de uso de tecnologias condizentes com o que existe no ambiente profissional e a não utilização de recursos mais interativos que propiciem aos aprendizes um estudo direcionado às suas necessidades específicas, promove a redução no interesse dos estudantes, dificultando a assimilação almejada e prejudicando o avanço nas habilidades relevantes à formação na área da Saúde.

A literatura mostra ainda a importância do uso de metodologias ativas em sala de aula como facilitadoras do aprendizado de Bioquímica, como, por exemplo, a técnica do "aprender-fazendo", na qual os alunos se tornam sujeitos do ensino, com livre expressão de sua criatividade por meio de jogos, gincanas, construção de modelos estruturais de moléculas e maquetes e/ou a dramatização de processos bioquímicos em sala de aula (Mitre et al, 2008), (Zierer e Assis, 2010).

É possível examinar na pesquisa de Oliveira et al (2007) que a utilização de uma metodologia educacional condizente com os objetivos almejados por uma disciplina de Bioquímica conseguiu promover o entusiasmo em seus alunos num curso de odontologia. Estes autores atribuíram essa motivação ao reconhecimento dos estudantes em observar a aplicação prática da disciplina e o importante envolvimento dela nas novas descobertas da ciência moderna, inclusive, chegando a promover alterações no mercado de trabalho.

Uma revisão sistemática da produção científica sobre estratégias didáticas utilizadas no ensino de Bioquímica em universidades brasileiras, publicados no período de 2004 a 2015, evidenciou que entre as atividades mais frequentes estavam a realização de atividade experimental (32,5\%), o uso de ferramentas didáticas variadas $(22,5 \%)$, o uso de Problem-based Learning (20\%), de tecnologias de informação (17,5\%) e o desenvolvimento de cursos $(7,5 \%)$. Com isso, observou-se que há grupos de professores trabalhando com diferentes estratégias didáticas buscando melhorar a assimilação discente (Silveira e da Rocha, 2016).

Neste contexto, a importância do estudo da bioquímica e as dificuldades pertinentes às características mais tradicionais aos processos educacionais em sua área de conhecimento têm incentivado a elaboração de propostas de ensino que objetivam 
VIII Congresso Brasileiro de Informática na Educação (CBIE 2019)

Anais do XXV Workshop de Informática na Escola (WIE 2019)

melhorar o seu processo instrucional envolvendo várias estratégias educacionais, algumas associadas com tecnologias mais interativas que fornecem maior autonomia aos discentes (Garrido et al, 2010).

Dentre estas perspectivas a utilização de uma metodologia de aprendizagem, associada a um tipo de software educacional "inteligente", foi empregada no ensino de graduação na disciplina de Bioquímica como uma abordagem instrucional inovadora na educação praticada na área da Saúde.

\section{Teoria da Aprendizagem Significativa (TAS)}

A Teoria da Aprendizagem Significativa entende que para acontecer o aprendizado de um conteúdo novo na estrutura cognitiva do ser humano adulto é preciso que este conteúdo seja associado a algum conhecimento anteriormente estabelecido na estrutura cognitiva do indivíduo (conceitos subsunçores), fazendo com que este novo conteúdo se torne um conhecimento significativo ao aprendiz. Ser significativo então corresponde ao conteúdo que foi armazenado de forma mais substantiva na estrutura mental do aprendiz e não aquele que foi memorizado, de maneira arbitrária e sem possuir relacionamento com outros conhecimentos que já estavam disponíveis na sua estrutura cognitiva (Ausubel, Novak e Hanesian, 1980).

De acordo com a circunstância que se encontre o aprendiz em relação aos novos conteúdos que devem ser assimilados, estes princípios serão empregados de maneira contínua e combinadas, a fim de atenderem as necessidades particulares de cada aprendiz frente a tais conteúdos. Por exemplo, na situação de um novo conceito ser assimilado por um aprendiz específico será importante, inicialmente, a compreensão mais substantiva de tal conceito, procurando estabelecer associações do novo conceito com os subsunçores disponíveis em sua estrutura cognitiva (princípio da Diferenciação Progressiva).

O princípio da Reconciliação Integrativa corresponde as reflexões envolvendo este novo conceito que está sendo assimilado, e será melhor integrado na estrutura cognitiva do aprendiz por meio do estabelecimento de mais associações com os subsunçores, melhorando sua ancoragem. Assim, por meio de análises e comparações novas relações serão estabelecidas entre aquilo que é novo e os subsunçores, promovendo a assimilação desejada através da interação dos dois princípios, a fim de melhor acomodar o novo conceito e fortalecer os subsunçores na estrutura cognitiva do aprendiz (subsunção pela TAS).

Para que essa aprendizagem ocorra é necessário o comprometimento responsável dos envolvidos diretamente no processo educacional, sendo pressupostos dois requisitos essenciais que direcionarão a postura e as ações dos discentes e docentes que objetivam trabalhar para que a aprendizagem significativa aconteça:

- A elaboração e uso de material potencialmente significativo aos aprendizes;

- A disposição proativa do aprendiz em relacionar o novo conteúdo à sua estrutura cognitiva, de maneira substantiva e não arbitrária (sem memorizar ou decorar simplesmente).

A TAS define dois tipos de significados que representam características expressivas aos objetivos almejados pela metodologia de aprendizagem que ela fundamenta, contribuindo ainda na indicação do estado da assimilação dos novos conteúdos pelos indivíduos interessados em aprender. 
VIII Congresso Brasileiro de Informática na Educação (CBIE 2019)

Anais do XXV Workshop de Informática na Escola (WIE 2019)

A expressão Significado Lógico faz referência às características inerentes ao conteúdo que se deseja assimilar, estando relacionada somente com os aspectos expressivos de sua própria natureza. Todavia, quando o aprendiz transforma o Significado Lógico em um conhecimento agregado à sua estrutura cognitiva torna-o Significado Psicológico. Essa mudança, de Significado Lógico para Significado Psicológico, é resultante da subsunção efetuada pelo aprendiz de maneira idiossincrática e retrata a situação da assimilação obtida (Ausubel, Novak e Hanesian, 1980).

A organização dos conceitos, ou itens a serem explorados por um conteúdo letivo, é fundamental ao êxito de sua assimilação significativa, colaborando na satisfação do primeiro requisito e facilitando no respeito da primeira hipótese da TAS. O emprego da técnica conhecida como Mapa Conceitual facilita essa organização, suportando todos os pressupostos da TAS e preparando os conceitos envolvidos em seus relacionamentos e hierarquias existentes.

A elaboração desse mapa promove a definição de sequências instrutivas hierarquicamente coerentes, fornecendo uma representação diagramática de fácil compreensão e manipulação das estruturas conceituais envolvidas, além da visão integrada do assunto abordado (Novak, 1998).

Geralmente, a elaboração do mapa conceitual é feita por especialistas responsáveis pelo ensino-aprendizagem de um determinado conteúdo (disciplina), sendo no mapa representada a expectativa de aprendizagem dos estudantes, além de seu compartilhamento entre todos os envolvidos no processo educacional. Esse compartilhamento é ampliado com a proposta de efetuar a aprendizagem significativa na disciplina de bioquímica, envolvendo a tecnologia do Sistema Tutor Inteligente conhecido como SAE (Sistema de Apoio Educacional).

Este software educacional (SAE) precisa do mapa conceitual para "aprender" como a disciplina está organizada e poder acompanhá-la empregando seus recursos interativos e "inteligentes" para auxiliar os estudantes a alcançarem a aprendizagem significativa em cada um de seus conceitos ou itens letivos que constituem a disciplina de bioquímica e estão representados no mapa conceitual (Rissoli, 2007).

\subsection{Sistema Tutor Inteligente (STI)}

A tecnologia dos Sistemas Tutores Inteligentes corresponde ao um software educacional que possui arquitetura modular, sendo a mais tradicional constituída de quatro módulos principais (Domínio, Aluno, Tutor e Interface), além de seu mecanismo de controle. Esse tipo de software é baseado em conhecimento e emprega técnicas provenientes da Inteligência Artificial, a fim de realizar análises e efetuar inferências sobre as diversas informações que são obtidas por seus diferentes módulos (Carbonell, 1970).

Os STIs mais tradicionais atuariam como um tutor individual de cada estudante que o utilizasse, trabalhando como ferramenta tecnológica mais voltada ao ensino, sem maiores preocupações com aspectos relativos à aprendizagem, que por sua vez ainda demandaria do acompanhamento contínuo e a colaboração de outros perfis participantes do processo educacional, como usuários do STI, sendo o caso dos docentes e de coordenadores pedagógicos.

Diante dessas demandas, e outras necessidades pertinentes a realidade da educação atual, é estabelecida uma classificação entre os STIs tradicionais e os mais modernos, que continuam atuando no ensino, mas também na assistência e na superação dos desafios pertinentes à aprendizagem personalizada e significativa. Yacef (2002), 
VIII Congresso Brasileiro de Informática na Educação (CBIE 2019)

Anais do XXV Workshop de Informática na Escola (WIE 2019)

classifica estes modernos STIs como Intelligent Teaching Assistant (ITA), enquanto que os mais tradicionais seriam somente tutores artificiais dos aprendizes.

Para esta autora (Yacef) o ITA possui mais recursos de assistência à aprendizagem dos estudantes, além da participação dos docentes como seus novos usuários, que colaboram na atuação do STI e na consolidação da "inteligência" desse assistente virtual de ensino e aprendizagem.

Em contrapartida, o ITA fornece dados a respeito da realidade de cada estudante ao professor, possibilitando o acompanhamento mais detalhado e em tempo real sobre o comportamento interativo dos aprendizes e seus respectivos desempenhos. O SAE é reconhecido como um ITA que amplia a participação de novos usuários por permitir que, além dos docentes, os monitores estudantis também sejam usuários do STI, acoplando mais um módulo em sua arquitetura, sendo ele denominado Monitoria (Rissoli, 2007).

Diante das características do ensino em bioquímica, a integração da tecnologia do SAE, para as ações educacionais do docente, possibilitaria um apoio significativo ao estudo em sala de aula e extraclasse, empregando tecnologia capaz de propiciar interação contínua no auxílio da assimilação dos conteúdos previstos no mapa conceitual pelos estudantes (Rissoli e Santos, 2013). O SAE precisa do mapa conceitual para "aprender" como a disciplina está organizada, podendo assim acompanhá-la empregando seus recursos interativos e 'inteligentes' para auxiliar os estudantes à alcançarem a aprendizagem significativa em cada um dos conteúdos que formam o domínio da disciplina Bioquímica.

\section{Experimento}

A metodologia proveniente da TAS foi aplicada a uma turma de graduação matriculada na disciplina Bioquímica, com carga horária de 60 horas semestrais e oferecida no primeiro período letivo dos cursos da área da Saúde em uma universidade brasileira.

Por se tratar de uma disciplina que pertence ao ciclo básico de conteúdos estudados pela maioria dos cursos de graduação na área da Saúde, as turmas eram bem heterogêneas e com $25 \%$ de sua carga horária semestral em laboratório (aulas práticas).

A docente responsável participou de um curso de extensão universitária antes do experimento, que abordava, principalmente, questões metodológicas da TAS e tecnologias de apoio educacional. Sua primeira atividade antes do experimento foi elaborar o Mapa Conceitual da disciplina, baseando-se nos objetivos e na ementa da disciplina Bioquímica. A Figura 1 mostra a representação do mapa conceitual elaborado.

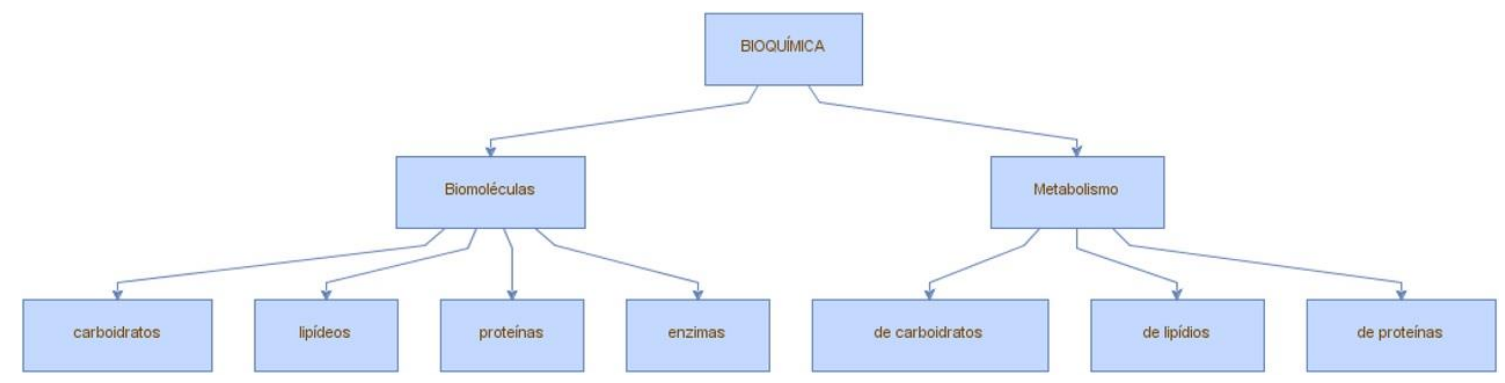

Figura 1 - Mapa Conceitual gerado pelo $\mathrm{SMC}^{1}$ da disciplina Bioquímica.

\footnotetext{
${ }^{1}$ Sistema de Mapas Conceituais (SMC) elaborado por trabalho de conclusão de curso em Ciência da Computação (Silva, Brum e Botelho, 2012).
} 
VIII Congresso Brasileiro de Informática na Educação (CBIE 2019)

Anais do XXV Workshop de Informática na Escola (WIE 2019)

Alguns outros dados relativos à disciplina e as atividades envolvendo o software educacional utilizado neste experimento (SAE) também foram definidos pela professora responsável (metadados), estando entre eles a quantidade de atividades e de questões interativas que cada estudante deveria estar realizando para a assimilação ser mais eficiente na concepção da professora, o número de possíveis visitas na monitoria estudantil disponível, o nível da aprendizagem significativa esperada em cada conteúdo para ser considerada satisfatória à assimilação entre outros dados mais operacionais.

Na segunda semana de aula os estudantes da turma foram apresentados ao SAE pela professora e a partir desse momento o acompanhamento da aprendizagem também passou a contar com o apoio desse STI. Com isso cada estudante poderia interagir com o SAE, além do período da aula, podendo reforçar os conteúdos estudados e alcançar a situação de aprendizagem satisfatória em cada um dos conteúdos letivos da disciplina que estavam previstos no mapa conceitual.

De acordo com a utilização do SAE por cada aprendiz, esse software prosseguia adquirindo conhecimento sobre os estudantes e fornecendo orientação pedagógica coerente e personalizada para as próximas ações dos aprendizes. Essa orientação objetivava facilitar a aprendizagem significativa, indicando caminhos mais condizentes com o perfil e a situação da aprendizagem de cada estudante acompanhado pelo SAE.

A orientação fornecida por este assistente virtual de ensino (SAE) era baseada na constatação dos termos linguísticos atribuídos para as variáveis linguísticas de esforço e desempenho, indicando para cada conceito presente no mapa conceitual da disciplina a situação da aprendizagem dos alunos (Rissoli e Santos, 2011). Entre os possíveis termos linguísticos a serem atribuídos para cada uma dessas variáveis era apurado o mais adequado por meio da aplicação da operação de maximo, caracterizada na Teoria dos Conjuntos Fuzzy como uma S-norma (Klir e Yuan, 1995). Essa apuração ocorria sobre os respectivos graus de pertinência que formavam o conjunto fuzzy relativo ao Significado Psicológico que cada aprendiz iria alcançando nos conceitos previstos no mapa conceitual.

Somente quando o aprendiz obtivesse todos os conceitos previstos no mapa conceitual da disciplina como satisfatórios na aprendizagem almejada e acompanhada pelo SAE, seria possível ao software calcular o índice de aprendizagem significativa que o estudante da disciplina alcançou. Esse índice corresponde a um fator de confiança fornecido pelo SAE que infere o quanto a aprendizagem de cada estudante foi significativa na disciplina Bioquímica, considerando os resultados específicos obtidos sobre cada conceito estudado e organizado no mapa conceitual.

\section{Resultados}

A realização do experimento possibilitou o acompanhamento personalizado de cada discente participante dessa disciplina de bioquímica, colaborando na atuação condizente com a realidade da turma pela docente nos encontros presenciais (aulas).

O nível de satisfação com o processo educacional envolvendo os discentes e a docente puderam ser confirmados por meio da pesquisa que todos foram convidados a participar no final do semestre letivo. Por meio dela foi solicitada a opinião de cada discente sobre as características da disciplina lecionada nessa forma experimental, os aspectos metodológicos observados e os recursos tecnológicos de apoio ao processo educacional, em que 19 (dezenove) alunos da turma participaram $(38,7 \%$ ) e todos indicaram somente aspectos positivos, quando questionados do porquê os recursos tecnológicos foram importantes em sua aprendizagem. Essa participação correspondeu a uma amostragem casual simples, com margem de erro de 18\% para um nível de confiança 
VIII Congresso Brasileiro de Informática na Educação (CBIE 2019)

Anais do XXV Workshop de Informática na Escola (WIE 2019)

de $95 \%$. Algumas das respostas fornecidas estão transcritas em poucas palavras, como solicitava esse questionamento da pesquisa:

"O SAE me disponibilizou um leque de perguntas relacionadas a bioquímica, fazendo assim um estudo muito agradável e bastante produtivo."

"Pois a partir destes a fixação do conteúdo não se tornou difícil."

"As questões do SAE, ajudavam a fixar melhor o que foi trabalhado e ensinado em sala de uma forma menos complexa."

"Pois é uma forma mais fácil de aprender."

É interessante também notar que $100 \%$ dos participantes da pesquisa dizem que o SAE colaborou no estudo e na aprendizagem. Dentre todos os estudantes que solicitaram orientação pedagógica para o SAE pouco mais de $84 \%$ relatam que este software os ajudou ou motivou na seara da aprendizagem em bioquímica, no qual pouco mais de $81 \%$ informaram que seguiram essas orientações para obter o êxito em seu próprio processo educacional.

Quando os alunos participantes dessa pesquisa são questionados se a disciplina teria atendido suas expectativas iniciais, 17 dizem que $\operatorname{sim}(89,5 \%)$ e estes ainda afirmam que a indicariam para outros colegas cursarem na forma em que está organizada (praticamente 90\%). Algumas dessas afirmações estão transcritas a seguir como depoimentos dos estudantes que passaram por este experimento:

"Indicaria, pois é um meio de estudo diferenciado e auxilia aqueles com mais dificuldades."

"Pois é mais fácil aprender a disciplina com esses recursos tecnológicos de apoio."

"Foi de extrema relevância e me ajudou a tirar dúvidas, foi muito mais além daquilo que podia esperar, me deu uma percepção em relação ao meu desempenho, considero que pode estimular e ajudar muito naquilo que é de extrema importância o conhecimento..."

"pois estes recursos tecnológicos ajudam na compreensão do conteúdo trabalhado em sala de aula."

"Porque o SAE me ajudou muito a entender bioquímica."

Essas constatações reforçam o ânimo da docente que está satisfeita com os resultados obtidos, assim como seus alunos e por isso solicitou que o SAE possa ser empregado na disciplina no próximo semestre letivo de bioquímica, de maneira regular e não mais experimentalmente.

\section{Conclusões}

A experiência que está sendo compartilhada por meio desse trabalho mostra a ação inicial partindo da docente envolvida com os desafios do processo educacional na disciplina de bioquímica, tradicionalmente, de difícil aprendizagem para estudantes que estão, geralmente, iniciando seu caminho de formação na área da Saúde.

Estando descontente com essa realidade, a professora foi buscar extensão para sua formação na Saúde e pôde conhecer a Teoria da Aprendizagem Significativa, além de tecnologias, ditas inteligentes, que poderiam fornecer suporte para a melhor tomada de 
VIII Congresso Brasileiro de Informática na Educação (CBIE 2019)

Anais do XXV Workshop de Informática na Escola (WIE 2019)

decisões didático-pedagógicas na condução de turmas de bioquímica, além de trabalhar as habilidades interativas e intelectuais em seus estudantes.

Essa melhor preparação possibilitou a elaboração de um novo ambiente educacional, que possuía novidades, em relação ao anterior mais tradicional, e permitia maior autonomia dos aprendizes, que eram acompanhados pelo Sistema de Apoio Educacional (SAE) e pelo docente. Esse novo ambiente fornecia a oportunidade de estudo mais interativo no período disponível dos estudantes, atentando, ainda, ao ritmo de aprendizagem individual que cada aluno da turma possuía.

Observando as respostas obtidas na pesquisa aplicada no final do período letivo foi possível confirmar alguns aspectos relativos a maior participação dos estudantes na disciplina, em que 16 alunos (pouco mais de 84\%), dos 19 participantes, classificaram a própria participação como ótima $(15,8 \%)$ ou boa $(68,4 \%)$. Somente 3 alunos $(15,8 \%)$ classificaram a própria participação como tendo sido pouca. Na observação da participação dos colegas de turma esses alunos indicaram que quase 69\% (13) aconteceu com maior intensidade, no qual pouco mais de 5\% classificou como ótima e $63 \%$ como boa.

A principal contribuição desse trabalho está na combinação das técnicas e métodos com os recursos tecnológicos que lhe sejam coerentes aos objetivos almejados, promovendo um ambiente mais justo para as diferentes realidades de cada aprendiz durante o estabelecimento individual de seu caminho de estudo e aprendizagem em bioquímica.

Dessa forma, a qualidade da aprendizagem e a satisfação com o processo educacional, em que quase $90 \%$ se dizem satisfeitos e que indicariam para um colega cursar esta disciplina neste novo formato, também confirmam essa contribuição, que envolveu aspectos inovadores em lecionar bioquímica respeitando princípios fundamentais da TAS, associados ao acompanhamento e as possibilidades de orientação sob medida de cada estudante por um software educacional 'inteligente' (SAE).

\section{Referências}

Albuquerque M. A. C.; Amorim A. H.C.; Rocha J.R.C.F.; Silveira L. M. F. G. e Neri D. F. M. (2012) Bioquímica como Sinônimo de Ensino, Pesquisa e Extensão: um Relato de Experiência. Revista Brasileira de Educação Médica. 36(1): 137-142.

Ausubel, D. P. (2000) The acquisition and retention of knowledge: A cognitive view. Dordrecht: Kluwer Academic.

Ausubel, D. P.; Novak, J. D. e Hanesian, H. (1980) Psicologia Educacional. Rio de Janeiro: Editora Interamericana.

Carbonell, J. R. (1970) AI and CAI: an artificial intelligent approach to computer- assisted instruction. IEEE Transactions on Man-Machine Systems, New York, 11(4): 190-202.

de Andrade, R. S. B.; Silva, A. F. da S. e Zierer, M. de S. (2017) Avaliação das dificuldades de aprendizado em Bioquímica dos discentes da Universidade Federal do Piauí. Revista de Ensino de Bioquímica, [S.1.], v. 15, n. 1, p. 24-39.

Garrido R. G.; Araújo, F. O.; Oliveira, T. H. e Garrido, F. S. R. G. (2010) O lugar da Bioquímica no processo de cuidar: visão de graduandos em Enfermagem. Revista Brasileira de Ensino em Bioquímica e Biologia Molecular. 8 (1): 30-35.

Gee, J. P. (2008) What video games have to teach us about learning and literacy, revised and updated. Basingstoke: Palgrave Macmillan.

Klir, J. e Yuan, B. (1995) "Fuzzy sets and fuzzy Logic - theory and applications", Upper Saddle River, Prentice-Hall PTR, 574p. 
VIII Congresso Brasileiro de Informática na Educação (CBIE 2019)

Anais do XXV Workshop de Informática na Escola (WIE 2019)

Mitre, S. M.; Siqueira-Batista, R.; Mendonça, J. M. G.; Pinto, N. M. M.; Meirelles, C. A. B. e Pinto-Porto, C. (2008) Metodologias ativas de ensino-aprendizagem na formação profissional em saúde: debates atuais. Cienc. Saúde Coletiva. 13(2):2133-2144.

Novak, J. D. (1998) Learning, creating, and using knowledge: Concept maps as facilitative tools in schools and corporations. New Jersey: Lawrence Erlbaum.

Oliveira, R. C. de; Monteiro, N. F.; Iano, F. G.; da Silva, T. L. e Buzalaf, M.A. (2010) Expectativas dos alunos quanto à importância da Bioquímica em sua carreira. Revista de Ensino de Bioquímica. Abr. 9;8(1):36-44.

Oliveira, R. C. de; Iano, F. G.; Silva, T. L. da e Buzalaf, M. A. F. (2007) Percepção dos Alunos do Curso de Odontologia de uma Universidade Brasileira em Relação à Importância da Disciplina de Bioquímica na sua Profissão. RBEBBM nº1 Artigo B.

Pinheiro T. D., da Silva J. A.; de Souza P. R.; do Nascimento M. M. e de Oliveira H. D. (2009) Ensino de Bioquímica para acadêmicos de Fisioterapia: visão e avaliação do discente. Revista de Ensino de Bioquímica. Fev. 25;7(1):25-35.

Regis, W. C. B.; Gonçalves, M. L. G. S. e Santoro, M. M. (2001) Avaliação das potencialidades de um curso prático de bioquímica na implementação do ensino teórico em cursos profissionalizantes. RBEBBM Especial 1.

Rissoli, V. R. V. e Santos, G. A. (2013) O Agente Pedagógico Animado MInA. In: Simpósio Brasileiro de Informática na Educação. Campinas (SP): SBC. 828-837p.

Rissoli, V. R. V. e Santos (2011) G. A. Um Assistente Inteligente Fuzzy no Acompanhamento da Aprendizagem Significativa. In: Congresso da Sociedade Brasileira de Computação, 2011, Natal. Porto Alegre: SBC.

Rissoli, V. R. V (2007) Uma proposta metodológica de acompanhamento personalizado para Aprendizagem Significativa apoiada por um Assistente Virtual de Ensino Inteligente. Porto Alegre: PGIE, 2007. Tese (Informática na Educação) UFRGS.

Schönborn, K. J. e Anderson, T. R. (2010) Bridging the educational research-teaching practice gap: foundations for assessing and developing biochemistry students' visual literacy. Biochem Mol Biol Educ. 38(5): 347-54.

Silva, M. L. M. P.; Brum, R. M. O. A e Botelho, T. N. M. (2012) Sistema de Mapas Conceituais. Brasília: UCB, 2012. Graduação (Ciência da Computação) UCB.

Silveira, J. T. e da Rocha, J. B. T. (2016) Produção científica sobre estratégias didáticas utilizadas no ensino de Bioquímica: uma revisão sistemática. Revista de Ensino de Bioquímica, [S.1.], v. 14, n. 3, p. 7-21.

Vieira, R. (2003) Fundamentos de Bioquímica: textos didáticos. Disponível em: $<$ https://www.ebah.com.br/content/ABAAABtO4AK/fundamentosbioquimicaricardo-vieira\#>. Acessado em: nov. 2018.

Yacef, K. (2002) Intelligent Teaching Assistant Systems. In: International Conference on Computers in Education. Proceedings. New Zeland: IEEE. v.1, p.136-140.

Yokaichiya, D. K.; Galembeck E. e Torres B. B. (2004) O que alunos de diferentes cursos procuram em disciplinas extracurriculares de bioquímica? São Paulo: Revista Brasileira de Ensino de Bioquímica e Biologia Molecular. 1:37-44.

Zierer, M. S. e Assis, R. C. (2010) A construção de modelos como estratégia para um ensino mais criativo nas disciplinas de bioquímica e biologia molecular. Diálogos \& Ciência (Online), v. 8, p. 1-14. 